\title{
A Precision Spraying Mission Assignment and Path Planning Performed by Multi-Quadcopters
}

\author{
Baihui Du ${ }^{1, *}$ \\ ${ }^{1}$ School of Automation Science and Electrical Engineering, Beihang University, Beijing, 100191, China \\ ${ }^{*}$ Corresponding author
}

\begin{abstract}
This paper proposes a hierarchal approach to solving a spraying mission assignment and path planning problem by using multi-quadcopters in an upland area. A mathematical model of the mission assignment and path-planning problem has been established. An inner-and-outer loop structure is employed in the hierarchal approach, in which the inner loop utilizes a genetic algorithm-based Travelling Salesman Problem (TSP) method while the outer loop uses a nonlinear programming method based on the optimal results given by the inner loop. In the end, simulation comparisons to a multi-TSP-based conventional approach have been carried out to illustrate the performance of the proposed approach.
\end{abstract}

Keywords-precision spraying; mission assignment; path planning; multi-quadcopters; Travelling Salesman Problem (TSP)

\section{INTRODUCTION}

Compared with the fixed-wing aircraft and helicopters, quadcopters are easier to utilize in the case of some specific tasks with excellent performance and a high level of autonomy [1][2]. As the level of autonomy in the agricultural industry improved with technical innovations, the usage of plantprotection quadcopters has become more common nowadays. The Chinese government takes great effort to accelerate agricultural modernization; one of the important concepts is precision agriculture, as in [3]. Precision agriculture, also called site-specific crop management, aims at optimizing returns on inputs while doing no harm the environment [4]. The precision agriculture is usually based on a geographic information system where all environmental data have been gathered. One of the critical components of the precision agriculture is the precision spraying. As many pesticides have more or less deleterious effects on the environment, precision spraying can not only optimize the pesticide usage but also reduce the damage to wildlife. The rise of precision agriculture coincides with the development of quadcopters [5]. Quadcopter is a powerful tool for the implementation of precision spraying mission, especially under complex geographic conditions that are extremely dangerous for human beings.

In the interdisciplinary field of precision protection and civil aviation, one important related problem is the multi-robots path-planning problem [6]. Currently, there are many types of research on the cooperative work and coordination of multiple robots. Reference [7] proposes an analytical path planning method for a system composed of multiple robots. The method takes into consideration the dynamic environment by utilizing local information. In [8], a cooperative coverage algorithm of multiple robots for the searching mission in an unknown grid environment is developed. This kind of research is mostly applied in reality, such as the floor cleaning, the mine detection, etc. There are also various studies concerning the path-planning problem of multi-UAVs. When this problem comes to multiUAVs, it becomes more complicated because UAVs move in three-dimensional space while mobile robots move only in two-dimensional space. Reference [9] establishes an A* optimization-based path-planning scheme for multi-UAVs. And reference [10] focuses on a specific mission to protect a group of ground vehicles followed by multi-UAVs. An optimal approach has been developed to solve the path planning and coordination of drones.

As the precision spraying places great emphasis on the specific target points rather than a simple coverage of the target area, it is instrumental to study point-by-point traversal problems. mTSP (multiple Travelling Salesmen Problem) is a chemetypical model for this kind of problem, as in [11]. Reference [12] defines mTSP as follows: given $n$ nodes and a cost metric associated, $m$ salesmen begin to take a tour from one depot and return to the depot in the end. Each node should be visited exactly once by one salesman. The goal is to determine the path for each salesman to minimize the total tour cost. Various research has been carried out on the modeling and the resolution of the mTSP model. As the mTSP belongs to NP-hard problems [13], it is efficient to employ a heuristic algorithm to obtain a near optimal result, as in [14]-[17]. Another approach is to decouple the mTSP problem into a set of TSP problems to improve computational accuracy. In this approach, it is flexible to build mathematical model according to the mission's specifications.

This paper mainly focuses on a practical engineering problem - a precision spraying mission under complex geographic regions performed by multi-quadcopters. The principal objective is to reduce working time on the premise of satisfying all the specifications of the spraying mission. A hierarchal approach is established to solve the mission assignment and path-planning problem. Then, the resolution is executed in this approach with a TSP-based inner loop and a nonlinear optimization-based outer loop. To illustrate the efficiency of this approach, simulation comparisons to a conventional approach have been conducted.

The remainder of this paper is organized as follows: Section II introduces the problem formulation. In section III, a conventional approach is firstly presented, then, a new hierarchal approach is developed. Section IV focuses on the 
simulation and comparison. A summary of the current study and future work is given in the end.

\section{MISSION DESCRIPTION}

This paper focuses on the precision spraying mission by using multiple plant-protection quadcopters. Figure 1 presents the topography and the target trees to be sprayed with pesticides as well as a schematic diagram of the mission.
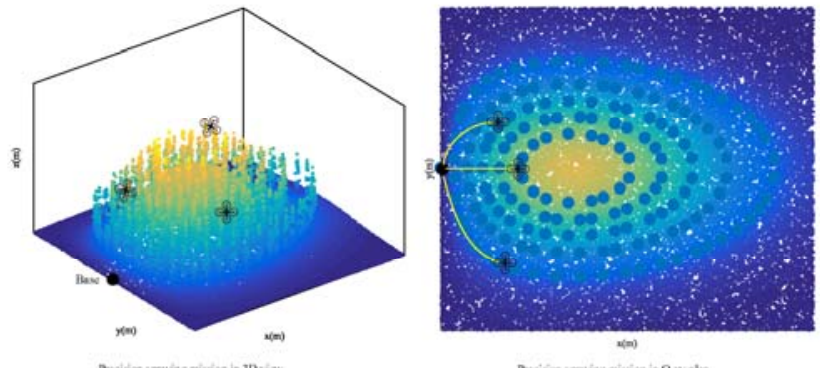

FIGURE I. A SCHEMATIC DIAGRAM OF THE MISSION

The black dot named Base is the base station where all the quadcopters take off and return back after mission completed. The target area is on a mountain so that its form is irregular. All the target trees are represented by blue points in the figure at the right. Both the location and the altitude of the target points are known. Quadcopters should spray pesticides above the tree, that is, each quadcopter begins to spray pesticides when it arrives at a target point and stops spraying when it flies to the next point. To satisfy the specifications of the mission, the following working principles for quadcopters are made:

1) Quadcopters move in three-dimensional space;

2) The total number of quadcopters is more than one and defined according to the situation;

3) Quadcopters are allowed to fail during the working process, such as crash because of sensor fault. In such failure, both mission assignment and path planning can be reconducted;

4) Once the battery capacity is low, the quadcopter should stop mission and return to the base. Mission assignment and path planning should be re-conducted;

5) All quadcopters take off from the base and return to the base after mission completed;

6) A quadcopter hover when it arrives at one target point and begin to spray pesticides, spraying time is predefined as $\tau$;

7) A quadcopter stops praying between two target points;

8) All the target points should be covered exactly once without missing.

Based on the principles above, given $m$ quadcopters and $n$ target trees to be covered, the study focuses on developing an automatic approach to divide a spraying mission into $m$ tasks and determine an optimal path for each quadcopter.

In this research, the primary measuring criterion is the working time to complete the mission. Let $T_{p}(1 \leq p \leq m)$ be the total working time of the $\mathrm{p}^{\text {th }}$ quadcopter. The objective is to minimize the maximum working time among $m$ tasks. The problem can be formulated as follows:

$$
\min \left(\max _{1 \leq p \leq m} T_{p}\right)
$$

It is evident that both the mission assignment scenarios and the path planning methods for each quadcopter may result in different working time. Thus, the core problem is transformed into an optimization problem:

\section{MATHEMATICAL APPROACHES AND OPTIMIZATION METHODS}

\section{A. A Conventional Approach}

As explained in Section II, the mission is that $m$ quadcopters should cover $n$ target points, and the optimization objective is to minimize the maximum working time $\max _{1 \leq p \leq m} T_{p}$.

A conventional approach is to formulate the optimization problem as a single-depot single-end mTSP model with time windows and additional constraints. The depot and the end locations in this mission refer to the base of quadcopters. The time window refers to the time associated with each target point during which the quadcopter sprays the pesticides, namely the spraying time $\tau$. The additional constraint is defined by the battery capacity of quadcopters, which is assumed to be identical for all quadcopters.

Let $V$ be the set of all target points, and $A$ be the set of edges. $C$ is a cost matrix associated with $A$. In our case, $C$ is a time matrix composed by two parts:

$$
C_{i j}=c_{i j}+c_{j}^{t w}
$$

where $c_{i j}$ is a conventional cost representing the flying time from the target point $i$ to the target point $j$, and $c_{j}^{t w}$ is a timedependent cost representing the spraying time $\tau$ of each quadcopter.

Let $T_{\text {lim }}$ be the longest working time of a quadcopter, which guarantees the quadcopter return to the base before the battery draining. In addition, define

$$
x_{i j p}=\left\{\begin{array}{l}
1, \quad \text { the } p^{t h} \text { quadcopter passed the edge }(\mathrm{i}, \mathrm{j}) \\
0,
\end{array}\right.
$$

Then, the mathematical model can be written as:

$$
\min \left(\max _{1 \leq p \leq m} T_{p}\right)
$$

where $T_{p}=\sum_{i=0}^{n} \sum_{j=0}^{n} \mathrm{C}_{i j} \cdot x_{i j p}$, s.t.

$$
\sum_{j=0}^{n} \sum_{p=0}^{m} x_{1 j p}=m
$$




$$
\begin{gathered}
\sum_{i=0}^{n} \sum_{p=0}^{m} x_{i 1 p}=m \\
\sum_{i=0}^{n} \sum_{p=0}^{m} x_{i j p}=1, i=2, \ldots, n \\
\sum_{j=0}^{n} \sum_{p=0}^{m} x_{i j p}=1, j=2, \ldots, n \\
\sum_{i=0}^{n} \sum_{j=0}^{n} \mathrm{C}_{i j} \cdot x_{i j p} \leq T_{\text {lim }}, p=1, \ldots, m
\end{gathered}
$$

where $\forall(i, j) \in A, p=1, \ldots, m, x_{i i p} \in\{0,1\}$.

In this model, (3) and (4) guarantee that all the target points can be visited, while (5) and (6) guarantee that each point is visited no more than once. (7) is the additional constraint representing the battery capacity limitation.

This modeling approach is, in fact, a mixture design of mission assignment and path planning. The advantage of this model is that all the specifications of the mission have been respected without any simplification. Equations (2) to (7) matches fully interpreted every aspect of the mission.

The completeness of the modeling, however, may result in low accuracy or reduced precision in the solution. MTSP is a typical NP-hard problem. Genetic Algorithm (GA) can be used to find a near optimal solution for this kind of problem. However, the GA-based optimization method often falls into a local optimum, especially in the case of considerable target points. Moreover, we have added time windows as well as an additional constraint in the model, which largely increase the computational complexity for the optimization. This is the original inspiration for developing an advanced approach to the mission.

In this paper, the resolution of the mTSP optimization employs an advanced GA proposed by Andras Kiraly and Janos Abonyi, see [18]. This algorithm has been proved to have a faster convergence rate and a more performant optimal solution compared with other algorithms.

\section{B. A hierarchical Approach and Corresponding Optimization Methods}

The conventional approach is less accurate especially in the case of a considerable number of target points because the mission assignment and the path planning problems are mixed in one optimization problem. To improve the performance of the optimization, it is intuitive that the mission assignment problem and the path-planning problem should be decoupled to reduce the computational complexity of the optimization process.

An advanced approach aims at pre-dividing target areas for each quadcopter and then planning a smooth path for the quadcopter. The basic idea is to develop a hierarchal design: the outer loop optimizes mission assignment schemes based on the calculation result of the inner loop, while the inner loop optimizes the working time of each quadcopter. In the end, the best solution will be saved.
Figure 2 presents the whole approach design in detail. First, to divide the target areas into $m$ circular sectors with center point located at the base. Then to use TSP modeling to determine an optimal path for each quadcopter and calculate the working time in each subarea, which is the inner loop. The optimization objective of the outer loop is to minimize the maximum working time among the $m$ quadcopters. The best solution will be obtained once the closed loop iterations finish.

One of the specialties of this approach is about the circularsector division. It is more common to use rectangular segment in this kind of mission, such as a pesticide-spraying mission in farmland, as in [19]. In our case, considering the fact that all the quadcopters take off from the same place as well as irregular paths in 3D space, the circular-sector division method can help strategically improve the optimization, as illustrated in figure 3 .

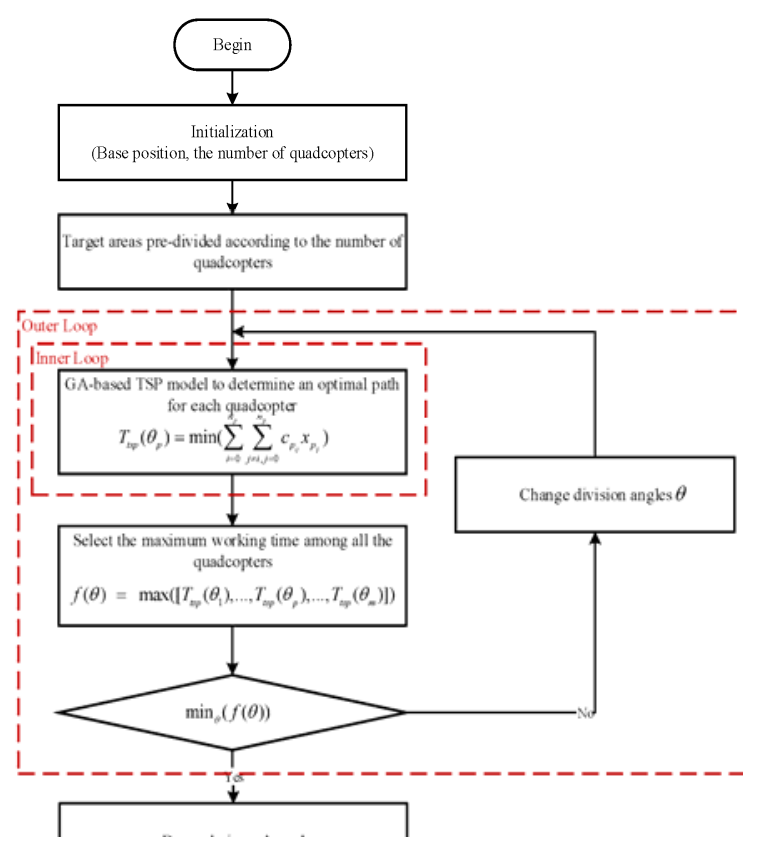

FIGURE II. FLOW CHART OF THE ADVANCED APPROACH

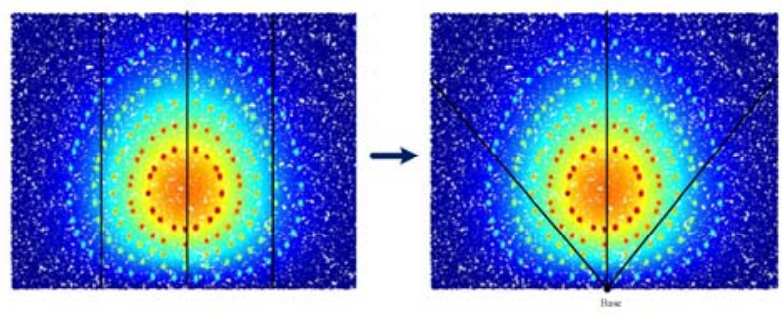

FIGURE III. CIRCULAR-SECTOR DIVISION METHOD

Let the angle of each sector be $\theta_{p}, p \in[1, m]$, and the corresponding sector be $S_{\theta_{p}}$. The optimization variable 
$\theta=\left[\theta_{1}, \ldots, \theta_{p}, \ldots, \theta_{m}\right]$. The objective of the outer loop optimization is a function of $\theta$ :

$$
\min _{\theta} f(\theta)
$$

where $f(\theta)=\max \left(\left[T_{t s p}\left(\theta_{1}\right) \ldots T_{t s p}\left(\theta_{m}\right)\right]\right)$ s.t.

$$
\begin{aligned}
& {[1, \ldots 1]_{1 \times m} \cdot \theta=\pi} \\
& 0 \leq \theta_{p} \leq \pi
\end{aligned}
$$

Hereafter a TSP-based path-planning method is given for the $\mathrm{p}^{\text {th }}$ quadcopter. Let $n_{p}$ be the number of target points in the sector $S_{\theta_{p}}$. Let $V_{p}$ be the set of all target points in this sector, and $A_{p}$ be the set of edges. $c_{p}$ is a cost matrix associated with $A_{p}$ where $c_{p_{i j}}$ represent the flying time of the $\mathrm{p}^{\text {th }}$ quadcopter from the target point $i$ to the target point $j$.

Similarly, $x_{p_{i j}}=\left\{\begin{array}{l}1, \quad \text { the } \mathrm{p}^{\text {th }} \text { quadcopter passed the edge }(\mathrm{i}, \mathrm{j}) \\ 0, \quad \text { the } \mathrm{p}^{\text {th }} \text { quadcopter didn't pass the edge }(\mathrm{i}, \mathrm{j})\end{array}\right.$

define

Then, the inner loop optimization objective $T_{t s p}\left(\theta_{p}\right)$ can be written as:

$$
T_{t s p}\left(\theta_{p}\right)=\min \left(\sum_{i=0}^{n_{p}} \sum_{j \neq i, j=0}^{n_{p}} c_{p_{i j}} x_{p_{i j}}\right)
$$

with

$$
0 \leq x_{p_{i j}} \leq 1, \quad \mathrm{i}, \mathrm{j}=0, \ldots, l_{p}
$$

$\sum_{i=0, i \neq j}^{l_{p}} x_{p_{i j}}=1, \quad \mathrm{j}=0, \ldots, l_{p}$

$\sum_{j=0, j \neq i}^{l_{p}} x_{p_{i j}}=1, \quad \mathrm{i}=0, \ldots, l_{p}$

$$
\sum_{i=0}^{n_{p}} \sum_{j \neq 1, j=0}^{n_{p}} c_{p_{i j}} x_{p_{i j}} \leq T_{l i m}
$$

where $\forall(i, j) \in A_{p}, x_{p_{i j}} \in\{0,1\}$.

The whole optimization problem is formulated as a hierarchal optimization approach where the inner loop optimization employs TSP model, and the outer loop optimization is a nonlinear minimization model with constraints. Both of the optimization methods are common enough to be easily implemented in applications.

The goal of this approach is to separate the path planning from the mission assignment and to improve performance and accuracy of the solution further. Simulation results and comparison with the conventional approach have been given in the next section.

\section{SIMULATION AND COMPARISON}

Comprehensive simulations have been carried out to illustrate the performance of the proposed approach. Also, a comparison between the conventional approach and the hierarchal approach has been given in this section. All the MATLAB simulations are conducted on a computer running Windows 7, with an i7 3.60GHz processor and 10GB RAM.

The target area is in an irregular form of $800 \mathrm{~m}^{2}$. The number of target points is $n=163$. The location and the altitude of the target points are supposed to be known. The number of quadcopters $m$ can be defined by the user. To begin with, consider a simple case where the spraying time $\tau$ is zero, the number of quadcopters $\mathrm{m}=3$ and the flying velocity is a constant with $V_{\text {fly }}=1 \mathrm{~m} / \mathrm{s}$.

Figure 4 is the mission assignment and path-planning result by the conventional approach. Figure 5 is the result by the hierarchal approach. The task assignment results are different by different approaches. The cumulative mission time of the first approach is $303.7 \mathrm{~s}$, and that of the second approach is $274.4 \mathrm{~s}$. It is clear that result of the hierarchal approach is more performant in the sense of mission time reduction and the smoothness of planned path.

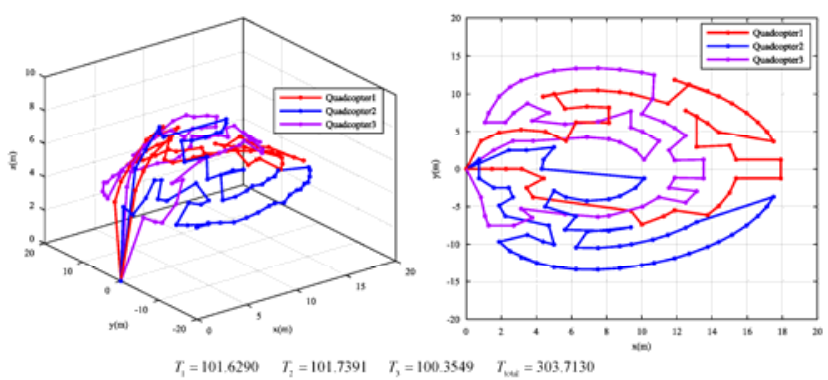

FIGURE IV. SOLUTION OF THE CONVENTIONAL APPROACH

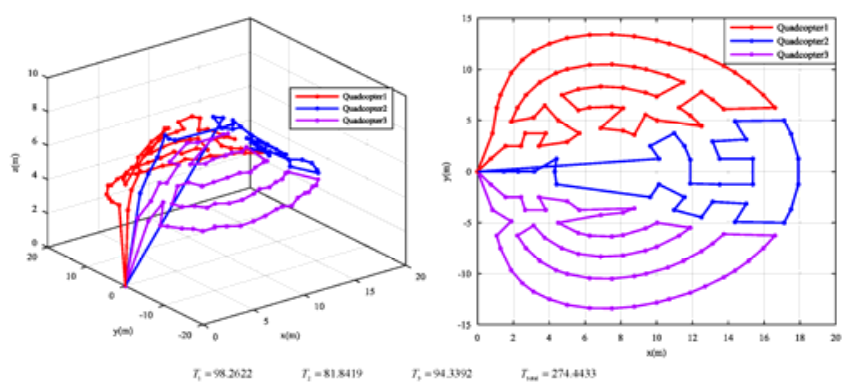

FIGURE V. SOLUTION OF THE HIERARCHAL APPROACH

It should be noted that the two approaches give only near optimal solution of the mission, which means that optimization results vary each time. The obtained mission time may have a slight difference. But the order of magnitude remains invariant. To make the results more persuasive, a comparison between 
the two approaches has been carried out, including 1) different numbers of quadcopters without accounting the spraying time $\tau$, and 2) fixed number of quadcopters with different spraying time $\tau$. Comparison results are given in TABLE I and II. $T_{\text {total }}$ is the cumulative time of $m$ quadcopters, and $\Delta T$ is the time gap between the maximum working time and minimum working time among the quadcopters.

TABLE I. COMPARISON RESULT BETWEEN THE TWO APPROACH WITH DIFFERENT NUMBERS OF QUADCOPTER

\begin{tabular}{|l|c|c|l|l|l|l|}
\hline \multicolumn{3}{|c|}{ Configurations } & \multicolumn{2}{c|}{ The conventional approach } & \multicolumn{2}{c|}{ The hierarchal approach } \\
\hline $\boldsymbol{m}$ & $\boldsymbol{n}$ & $\boldsymbol{\tau}$ & \multicolumn{1}{|c|}{$\boldsymbol{T}_{\text {total }}$} & $\Delta \boldsymbol{T}$ & $\boldsymbol{T}_{\text {total }}$ & $\Delta \boldsymbol{T}$ \\
\hline 3 & 163 & 0 & 303.7130 & 1.3842 & 274.4433 & 16.4203 \\
\hline 4 & 163 & 0 & 340.2202 & 0.1536 & 289.9594 & 25.8544 \\
\hline 5 & 163 & 0 & 368.5407 & 2.2836 & 309.1263 & 23.5434 \\
\hline
\end{tabular}

TABLE II. COMPARISON RESULT BETWEEN THE TWO APPROACH WITH DIFFERENT SPRAYING TIME

\begin{tabular}{|l|c|c|l|l|l|c|}
\hline \multicolumn{3}{|c|}{ Configurations } & \multicolumn{2}{c|}{ The conventional approach } & \multicolumn{2}{c|}{ The hierarchal approach } \\
\hline $\boldsymbol{m}$ & $\boldsymbol{n}$ & $\boldsymbol{\tau}$ & \multicolumn{1}{|c|}{$\boldsymbol{T}_{\text {total }}$} & $\Delta \boldsymbol{T}$ & $\boldsymbol{T}_{\text {total }}$ & $\Delta \boldsymbol{T}$ \\
\hline 4 & 163 & 1 & 510.7437 & 0.6482 & 451.4718 & 23.3322 \\
\hline 4 & 163 & 3 & 842.2575 & 3.0639 & 789.0572 & 57.8797 \\
\hline 4 & 163 & 5 & 1183.0214 & 0.1895 & 1124.7000 & 72.7022 \\
\hline
\end{tabular}

As shown in TABLE I, the total mission time is much reduced by the hierarchal approach. The working time gap of this approach is larger than that of the conventional approach. It can be ameliorated by modifying the optimization objective in (8) to

$$
\left.f(\theta)=\max \left(\left[T_{t s p}\left(\theta_{1}\right) \ldots T_{t s p}\left(\theta_{m}\right)\right]\right)-\min \left(\mid T_{t s p}\left(\theta_{1}\right) \ldots T_{t s p}\left(\theta_{m}\right)\right]\right)
$$

The spraying time $\tau$ will decrease the accuracy of the optimization resolution. As shown in table 2, optimization differences by two approaches diminish as values of $\tau$ augment. As the optimization objective is composed of two parts: the flying time and the spraying time. The spraying time is a constant value, while the optimization only reduces the flying time. And when the spraying time is dominant, the optimization effect will be diminished.

\section{CONCLUSION}

This paper proposes a hierarchal approach to solve the mission assignment and the path-planning problem of upland precision spraying mission. Both theoretical and computational comparisons have been conducted with the mTSP-based conventional approach. In the end, a serial of simulations has illustrated the performance and efficiency of this approach. This approach mainly solves the automatic decision problem when we use quadcopters to spray pesticides on specific targets in a complex geographic condition, and the objective is to reduce the mission time.

For the future studies, an interactive platform will be established based on our current research. Given the number of quadcopters and data map of target areas, the mission assignment and the path planning result can be determined automatically. Moreover, the optimization may further extend to a dynamic approach which enables real-time decisionmaking.

\section{REFERENCES}

[1] R.Austin, "Unmanned aircraft systems: UAVs design, development and deployment," John Wiley \& Sons, 2011.

[2] Q.Quan, "Introduction to multicopter design and control," Springer, 2017.

[3] "The future of agriculture," The Economist, 2016, in press.

[4] A.McBratney, B.Whelan, T.Ancev, "Future directions of precision agriculture,” Precision Agriculture, vol.6, pp.7-23,2005

[5] I.Schmal, G.David, B.Dingus and C.Reinholtz, "Development and application of an autonomous unmanned aerial vehicle for precise aerobiological sampling above agricultural fields," Journal of Field Robotics, vol.25, no.3, pp.133-147, 2008.

[6] C.Zhang and J.Kovacs, "The application of small unmanned aerial systems for precision agriculture: a review,"Precision Agriculture, vol.13, no.6, pp.133-147, 2012.

[7] W. Yu, J. Peng, X. Zhang and K. Lin, "A Cooperative Path Planning Algorithm for a Multiple Mobile Robot System in a Dynamic Environment," International Journal of Advanced Robotic Systems, 2014.

[8] Z. Butler, A. Rizzi and R. Hollis, "Cooperative coverage of rectilinear environments," in Proceedings of IEEE International Conference on Robotics and Automation, San Francisco, pp. 2722-2727, 2000.

[9] J.Zhao and J.Zhao, "Path Planning of Multi-UAVs concealment attack based on new A* method," 2014 sixth International Conference On Intelligent Human-Machine Systems And Cybernetics, pp. 401-404, 2014.

[10] X.Ding, A.Rahmani, and M.Egerstedt, "Multi-UAV convoy protection: an optimal approach to path planning and coordination," IEEE Transactions On Robotics, vol. 26, no. 2, April 2010.

[11] D.L.Applegate, R.E. Bixby, V.Chvátal and W.J. Cook, "The traveling salesman problem: a computational study (Princeton in applied mathematics)," Princeton University Press, 2007.

[12] T.Bektas, "The multiple traveling salesman problem: an overview of formulations and solution procedures," Omega, vol.34, no.3, pp.209-219, 2006 .

[13] M.Gary, D.Johnson, "Computers and intractability: a guide to the theory of NP-hard completeness," W. H. Freeman, 1979.

[14] R.A.Russell, "An effective heuristic for the m-tour traveling salesman problem with some side conditions." Operations Research, vol.25, no.3, pp.517- 524, 1977.

[15] A.Király and J.Abonyi, "Optimization of multiple traveling salesmen problem by a novel representation based genetic algorithm," Proceedings of the 10th International Symposium of Hungarian Researchers on Computational Intelligence and Informatics. Budapest, Hungary: IEEE Press, pp.315-326, 209.

[16] A.E.Carter and C.T.Ragsdale, "A new approach to solving the multiple traveling salesperson problem using genetic algorithms," European Journal of Operational Research, vol.175, no.1, pp.246-257, 2006.

[17] A.Singh and A.S.Baghel, "A new grouping genetic algorithm approach to the multiple traveling salesperson problem," Soft Computing - A Fusion of Foundations, Methodologies and Applications, vol.13, no.1, pp.95-101, 2009.

[18] A.Király and J.Abonyi, “ A novel approach to solve multiple traveling salesmen problem by genetic algorithm," Studies in Computational Intelligence, pp.141-151, 2010.

[19] Y.Luo, J.Yan, Z.Y.Zhao, S.Yang and Q.Quan, "A pesticide spraying mission assignment performed by multi-quadcopters and its simulation platform establishment," in Proceedings of 2016 IEEE Chinese Guidance, Navigation and Control Conference, pp.1980-1985, 2016. 